\title{
OPEN Lack of gamma delta T cells ameliorates inflammatory response after acute intestinal ischemia reperfusion in mice
}

Dominik Funken $^{1,7}, Y_{i} Y_{u^{2,7}}$, Xiaoyan Feng ${ }^{2,6}$, Tawan Imvised ${ }^{2}$, Faikah Gueler ${ }^{3,9}$, Immo Prinz $^{4,5}$, Omid Madadi-Sanjani², Benno M. Ure ${ }^{2}$, Jochen F. Kuebler ${ }^{2,8}$ \& Christian Klemann ${ }^{1,2,8 凶}$

T-cells have been demonstrated to modulate ischemia-reperfusion injury (IRI) in the kidney, lung, liver, and intestine. Whereas most T-cell subpopulations contribute primarily to the antigen-specific effector and memory phases of immunity, $\gamma \delta$-T-cells combine adaptive features with rapid, innatelike responses that can place them in the initiation phase of immune reactions. Therefore, we aimed to clarify the role of $\gamma \delta$-T-cells in intestinal IRI. Adult wild-type (WT) and $\gamma \delta$-T-cell-deficient mice were subjected to acute intestinal IRI. Gene expression of pro-inflammatory cytokines and influx of leukocyte subpopulations in the gut were assessed by qPCR and flow cytometry. Serum transaminases were measured as an indicator of distant organ IRI. Intestinal IRI led to increased influx of neutrophils, pro-inflammatory cytokine expression and LDH/ALT/AST elevation. Selective deficiency of $\gamma \delta$-Tcells significantly decreased pro-inflammatory cytokine levels and neutrophil infiltration in the gut following IRI compared to controls. Furthermore, $\gamma \delta$-T-cell deficiency resulted in decreased LDH and transaminases levels in sera, indicating amelioration of distant organ injury. Increasing evidence demonstrates a key role of T-cell subpopulations in IRI. We demonstrate that $\gamma \delta$-T-cell deficiency ameliorated pro-inflammatory cytokine production, neutrophil recruitment and distant organ injury. Thus, $\gamma \delta$-T-cells may be considered as mediators contributing to the inflammatory response in the acute phase of intestinal IRI.

Ischemia/reperfusion injury causes significant morbidity and mortality and occurs in a plethora of clinical conditions such as organ transplantation, resection, trauma, and septic- or hemorrhagic shock. In order to minimize the negative effects of IRI, the complex pathogenesis has to be elucidated. Inflammation has been identified as one key factor. Initially, tissue facors and the innate immune system were thought to mediate the inflammatory response following IRI. However, emerging data demonstrated a role of T cells as mediators of $\mathrm{IRI}^{1-6}$. A number of studies firmly established conventional $\alpha \beta \mathrm{T}$ cells as contributors to IRI in organs such as the liver and kidney. In intestinal IRI, however, the role of T cells remains controversial. Studies with SCID mice, which lack all T- and B-cells, have implicated an amelioration of intestinal IRI by a lack of T cells ${ }^{7,8}$. However, we have recently demonstrated that selective deficiency of conventional $\alpha \beta \mathrm{T}$ cells did not impact the damage in intestinal IRI ${ }^{9}$. Therefore, we hypothesized that the lack of $\gamma \delta$ T cells might be responsible for the observed amelioration of IRI in SCID mice. $\gamma \delta$ T cells are a unique T cell subset and among the very first T cells to develop in all vertebrates ${ }^{10-13}$. In the circulation, $\gamma \delta$ T cells represent only a minor fraction of T cells, but tissue-resident epithelial $\gamma \delta$ T cells represent the major T cell population in the epithelium of the gut ${ }^{14} . \gamma \delta \mathrm{T}$ cells combine features of the adaptive immune system, such as undergoing thymic selection and expression of a $\mathrm{T}$ cell receptor, with features of the innate immune system such as the capacity for pattern- or danger signal recognition in order

${ }^{1}$ Department of Pediatric Pneumology, Allergy and Neonatology, Hannover Medical School, Carl-Neuberg-Straße 1, 30625 Hannover, Germany. ${ }^{2}$ Department of Pediatric Surgery, Center of Surgery, Hannover Medical School, Hannover, Germany. ${ }^{3}$ Department of Nephrology, Hanover Medical School, Hannover, Germany. ${ }^{4}$ Institute of Immunology, Hannover Medical School, Hannover, Germany. ${ }^{5}$ Institute of Systems Immunology, University Medical Center Hamburg-Eppendorf, Hamburg, Germany. ${ }^{6}$ Present address: Department of Pediatric Surgery, University of Leipzig Medical Center, Leipzig, Germany. ${ }^{7}$ These authors contributed equally: Dominik Funken and Yi Yu. ${ }^{8}$ These authors jointly supervised this work: Jochen F. Kuebler and Christian Klemann. ${ }^{9}$ Faikah Gueler is deceased. ${ }^{\varpi}$ email: Klemann.Christian@mh-hannover.de 
to initiate rapid immune responses ${ }^{15,16}$. Of note, in IRI models of kidney and brain $\gamma \delta$ T cells have been shown to aggravate ischemic injury by generating chemotactic signals for peripheral myeloid cells such as neutrophils and monocytes ${ }^{17,18}$. Thus, in the study at hand, we aimed to clarify the role of $\gamma \delta$ T cells in intestinal IRI. Utilizing a mutant mice strain which selectively lacks $\gamma \delta$ T cells, we demonstrate that deficiency of this conserved lymphocyte population results in an amelioration of the inflammatory response in the first hours of acute intestinal IRI.

\section{Materials and methods}

Animals and model of intestinal IRI. All procedures were approved by the local animal welfare committee at the University of Veterinary Medicine Hannover (permit number 42502-04-12/0769). All methods in the study were carried out in accordance with the Helsinki guidelines and declaration or any other relevant guidelines. Furthermore, the study was carried out in compliance with the ARRIVE guidelines.

For all experiments, 4 weeks old male C57BL/6 J wild-type (WT) and mice deficient of $\gamma \delta$ T cells (B6.129P2)$\mathrm{T} c r d^{\text {tm1Mom }}$ ) (mean body-weight $15 \mathrm{~g}$ ) were used ${ }^{19}$. In brief, in B6.129P2-Tcrd ${ }^{\text {tm1Mom }}$ mice the gamma-delta cell receptor expression in all adult lymphoid and epithelial organs is deficient, while the alpha-beta T-cell lineage show unaffected development.

In order to reduce a possible influence of gut microbiota, all animals were housed in the same room of the facility and littermates were used. Intestinal IRI was induced as previously described ${ }^{20}$. In brief, mice were anesthetized with a combination of Ketamine $(100 \mathrm{mg} / \mathrm{ml}$, Albrecht, Germany) and Xylazine (2\%, Bayer HealthCare, Germany) by i.p. injection. Normothermia was maintained, oxygen supplemented, and for prevention of dehydration, $4 \mathrm{ml} / \mathrm{kg} / \mathrm{h}$ isotonic saline was injected subcutaneously. Ischemia was induced by clamping the superior mesenteric artery for 30 min with spring-loaded bulldog microvascular clamps (Aesculap, Germany) after midline laparotomy. The celiac trunk was preserved during the whole procedure, to reduce ischemic injury of the liver. Observation of pulsatile mesenteric flow was confirmed before closing the abdominal cavity confirmed reperfusion. After $4 \mathrm{~h}$ of surgery, animals were sacrificed, and small intestinal tissue and serum were collected. Previous studies have presented a highly variable degree of injury, following IRI in rodents, in the histology. Different segments of the intestine seem to be more or less sensitive for ischemic injury ${ }^{21}$. Therefore, histological quantification of the intestinal injury was not performed. Aged matched animals undergoing the same procedure except for clamping of the mesenteric artery served as controls in every experiment.

mRNA extraction and assessment of cytokine expression by quantitative reverse transcription-polymerase chain reaction (qPCR). mRNA extraction and assessment of cytokine expression by quantitative PCR was performed as previously described ${ }^{20,21}$. Briefly, total RNA was extracted from lysed gut tissue using RNeasy Mini Kit (Qiagen, Venlo, The Netherlands). A high-capacity RNA-to-complementary DNA Kit (Applied Biosystems, Foster City, CA) was used for complementary DNA preparation. All transcripts were assessed by QuantiTect Primer Assay (Qiagen) and Maxima SYBR Green/Rox qPCR MasterMix (Thermo Scientific, Waltham, MA) on an Applied Biosystems StepOnePlus Real-Time PCR System (Life Technologies, Carlsbad, CA). Relative gene expression values were normalized to Actin, glyceraldehyde-3-phosphate dehydrogenase, and Hypoxanthine-guanine phosphoribosyltransferase as housekeeping genes.

Isolation of intestinal leukocytes and flow cytometry. Intraepithelial leukocytes (IEL) and lamina propria leukocytes (LPL) were isolated and processed as previously described ${ }^{20,21}$. Briefly, intraepithelial leukocytes were isolated by incubating cleansed and mechanically homogenized and incubated in PBS with $10 \%$ fetal calf serum (FCS) (PAA Laboratories, Cölbe, Germany) in supplemented with 5 mM EDTA (Applichem, Darmstadt, Germany) on an orbital shaker. Subsequently, lamina propria cells were isolated after digestion with $100 \mu \mathrm{g} / \mathrm{mL}$ Liberase (Roche, Basel, Switzerland) and enriched by discontinuous 40\%/70\% Percoll (GE Healthcare, Buckinghamshire, United Kingdom) density gradient centrifugation. Estimation of absolute cell numbers and flow cytometric analysis were performed according to standard protocols as described previously ${ }^{21,22}$. Briefly, fixable Viability Dye (eBioscience, Santa Clara, CA) was used to exclude dead cells. Unspecific binding was prevented by incubation with anti-FcgRII/III mononuclear antibody (Clone: 2.4G2; Biolegend, San Diego, CA). Specific stainings were performed with the following antibody clones and conjugated fluorochromes (all eBiosciences), for T cells: CD45-30-F11-eFluor450, CD3e-145-2C11-PerCP-Cy5.5, CD4-RM4-5-APC-eFluor780, CD8a-53-6.7-FITC, TCR $\beta$-H57-597-APC, TCR $\gamma \delta$-eBioGL3-PE, and for myeloid cells CD45-30-F11-eFluor450 CD11b-M1/70-APC-efluor780, CD11c-N418-PerCP-Cy5.5, Ly6-G-1A8-PE-Cy7, Ly-6C-HK1,4-PE. Samples were acquired on a FACS Canto II flow cytometer with BD FACSDiva software (BD Biosciences, Becton, Dickinson and Company, USA, Version 8.0.1, https://www.bdu.edu/flow-cytometry/files/2010/10/BDFACSDiva SoftwareReferenceManual.pdf) and analyzed with KALUZA software (Beckman Coulter, Brea, USA, Version: 1.5.20365.16139, https://www.beckman.de/resources/videos/products/kaluza-software-performance). Myeloid subpopulations were assessed as described by Rose et al. ${ }^{23}$. and T cells as previously described by our group ${ }^{21}$.

Assessment of liver enzymes in serum. Lactatdehydrogenase (LDH), Alanine transaminase (ALT) and aspartate transaminase (AST) were measured by using an AU 400 Olympus Analyzer (Olympus, Tokyo, Japan) as previously described ${ }^{24}$.

Statistics. One-way analysis of variance with a Tukey's multiple comparisons test was performed with GraphPad Prism software, v6.0 (GraphPad Software, San Diego, CA, USA). Data are displayed as mean and SD with $\mathrm{p}<0.05$ considered as statistically significant. 


\section{Results}

Ameliorated leukocyte influx following IRI in $\gamma \delta$ T cell deficient mice. In order to elucidate the role of gamma delta $\mathrm{T}$ cells in acute intestinal IRI, we performed flow cytometric $\mathrm{T}$ cell phenotyping of lymphocytes isolated from the gut of WT mice and mice lacing $\gamma \delta$ T cells. Assessment of total cell numbers showed an increase of leukocytes following IRI in the gut with a significantly reduced number of leukocytes in animals deficient of $\gamma \delta$ T cells compared to WT animals (Fig. 1A). As $\gamma \delta$ T cells represent the major lymphocyte population among the IEL, overall lymphocyte numbers in the gut were slightly but statistically significantly reduced in animals deficient of $\gamma \delta$ T cells compared to WT (Fig. 1B). Conceivably, Tcrd ${ }^{\text {tmIMom }}$ mice displayed a significant decrease in their overall T cell fraction as compared to WT animals due to their lack of $\gamma \delta \mathrm{T}$ cells, especially in the IEL fraction (Fig. 1C). IRI did not impact the fraction of $\alpha \beta$ or $\gamma \delta$ T cells in each compartment (Fig. 1D,E).

Neutrophil influx is decreased $\gamma \delta T$ cell deficient mice. Neutrophils have been shown to be pivotal in mediating acute tissue damage in other organ models of IRI, while monocytes/macrophages extend the immediate injury $y^{25,26}$. Assessing the fraction of neutrophils and macrophages by flow cytometry revealed a massive influx of Ly6 $6 \mathrm{G}^{+}$neutrophils in the intestinal epithelium and, to a lesser extent, into the lamina propria (Fig. 2A). Statistical analysis of group data showed a diminished influx of neutrophils into the gut of $\gamma \delta$ T cell deficient mice compared to WT animals (Fig. 2B).

Diminished up-regulation of pro-inflammatory cytokines following IR in $\gamma \delta T$ cell deficient mice. An inflammatory response detrimental to the affected organ is a key feature of IRI. In order to find possible effects due to a lack of $\gamma \delta$ T cells in regard to the degree of inflammation, we assessed the expression of proinflammatory cytokines in the gut tissue by qPCR. Both chemokines CXCL1/KC and CXCL2/MIP-2 are major neutrophil attractants and mostly secreted by monocytes and macrophages. The archetypical pro-inflammatory cytokines IL- 6 and TNF- $\alpha$, are secreted by both T cells and macrophages, in order to regulate the inflammatory reaction in the acute phase response. Gene expression of CXCL1/KC, CXCL2/MIP-2, IL-6, TNF- $\alpha$, as well asIL-17A, were all upregulated following IRI in the gut (Fig. 3). Compared to WT mice, $\gamma \delta$ T cell deficient mice demonstrated a less pronounced up-regulation of these pro-inflammatory cytokines after IRI (Fig. 3).

Ameliorated far distant organ injury in animals deficient of $\gamma \delta \mathrm{T}$ cells. To test for a possible systemic effect after intestinal IRI, we measured ALT and AST levels in sera as markers of distant organ injury. Our results demonstrate a significant up-regulation of both enzymes following intestinal IR, which was less pronounced in $\gamma \delta$ T cell deficient mice (Fig. 4).

\section{Discussion}

Intestinal IRI is a significant clinical challenge with complex, multifactorial, and incompletely understood pathophysiology, but inflammation is known to be a common feature in different organ models. Recently, T cells gained interest as perpetuators of inflammation in a number of models of IRI ${ }^{1-6}$. Interestingly, studies in SCID mice, which lack all T and B cells, showed ameliorated intestinal IRI and thus presented convincing evidence that lymphocytes are involved in the pathogenesis. However, our previous study demonstrates that $\alpha \beta \mathrm{T}$ cells, the largest and conventional fraction carrying the $\mathrm{T}$ cell receptor, do not impact the acute phase of intestinal IRI and, therefore, may be considered as 'innocent bystanders'. Here, we extend these findings by demonstrating significant protection in $\gamma \delta$ T cell-deficient mice compared with wild-type mice in the early phase of intestinal IRI.

Intestinal mucosal lymphocytes, which include IEL and LPL, are pivotal in the mucosal immune system, especially by providing immune surveillance of the epithelium ${ }^{14}$. Most of the IEL and LPL are T cells, which carry either the conventional $\alpha \beta$ TCR or a $\gamma \delta$ TCR. In all vertebrates, $\gamma \delta$ T cells are the very first T cells to develop in the thymus but represent only a minor fraction of the circulating $\mathrm{T}$ cells in the body ${ }^{10}$. However, in epithelial tissue and especially in the gut, $\gamma \delta$ T cells are present in much higher numbers, sometimes exceeding the number of conventional $\alpha \beta \mathrm{T}_{\text {cells }}{ }^{14}$. These highly conserved $\gamma \delta \mathrm{T}$ cells have been shown to play an important role in immune homeostasis of the gut, represent a first layer of defense, and protect from intestinal inflammation in a murine model of inflammatory bowel disease ${ }^{27}$.

Consistent with previous data from our ${ }^{9}$ and other groups ${ }^{28}$, we show that roughly half of the $\mathrm{T}$ cells among the IEL carry the $\gamma \delta$ TCR and that the $\gamma \delta$ T cell fraction is lower in the lamina propria (Fig. 1). Conceivably, mice lacking $\gamma \delta \mathrm{T}$ cells had significantly fewer absolute lymphocyte numbers in the gut epithelium compartment (Fig. 1). The same effect was seen also in the lamina propria, but did not reach significance (Fig. 1). Importantly, IRI did not impact lymphocyte number or distribution in the gut in WT or $\gamma \delta$ T cell deficient mice following IRI in our model (Fig. 1), which confirms our previous data in a $\alpha \beta$ T cell deficient intestinal IRI model ${ }^{9}$. These findings are in line with previous works demonstrating no or only minor differences regarding lymphocyte numbers in the gut after $1-3 \mathrm{~h}$ of intestinal IRI ${ }^{7,29,30}$. Therefore, the pronounced increase of leukocytes into the intestinal epithelium as well as into the lamina propria (Fig. 1) is due to an influx of myeloid cells with neutrophils representing the largest fraction (Fig. 2). Correspondingly, we observed an upregulation of CXCL1/KC, CXCL2/MIP-2, as major neutrophil attractants, as well as IL- 6 and TNF- $\alpha$, as archetypical pro-inflammatory cytokines in the gut after intestinal IRI (Fig. 3) ${ }^{31}$. We also observed a highly significant reduction of the expression of IL-17A following IR in mice lacking gd T cells (Fig. 3). Innate lymphoid cells have previously demonstrated to be the major producer of IL-17A in intestinal IRI and lack of IL-17 ameliorated intestinal IRI ${ }^{32,33}$. Our data conceivably supports this notion by demonstrating that the lack of the major producers of IL-17A also ameliorates intestinal IRI.

Strikingly, lack of $\gamma \delta$ T cells resulted in a diminished leukocyte influx into the gut (Fig. 1), which we show to be caused by a reduced neutrophil influx (Fig. 2). In the corresponding assessment of cytokines, $\gamma \delta \mathrm{T}$ cell deficient mice demonstrated a less pronounced, but not completely abrogated, up-regulation of the assessed 


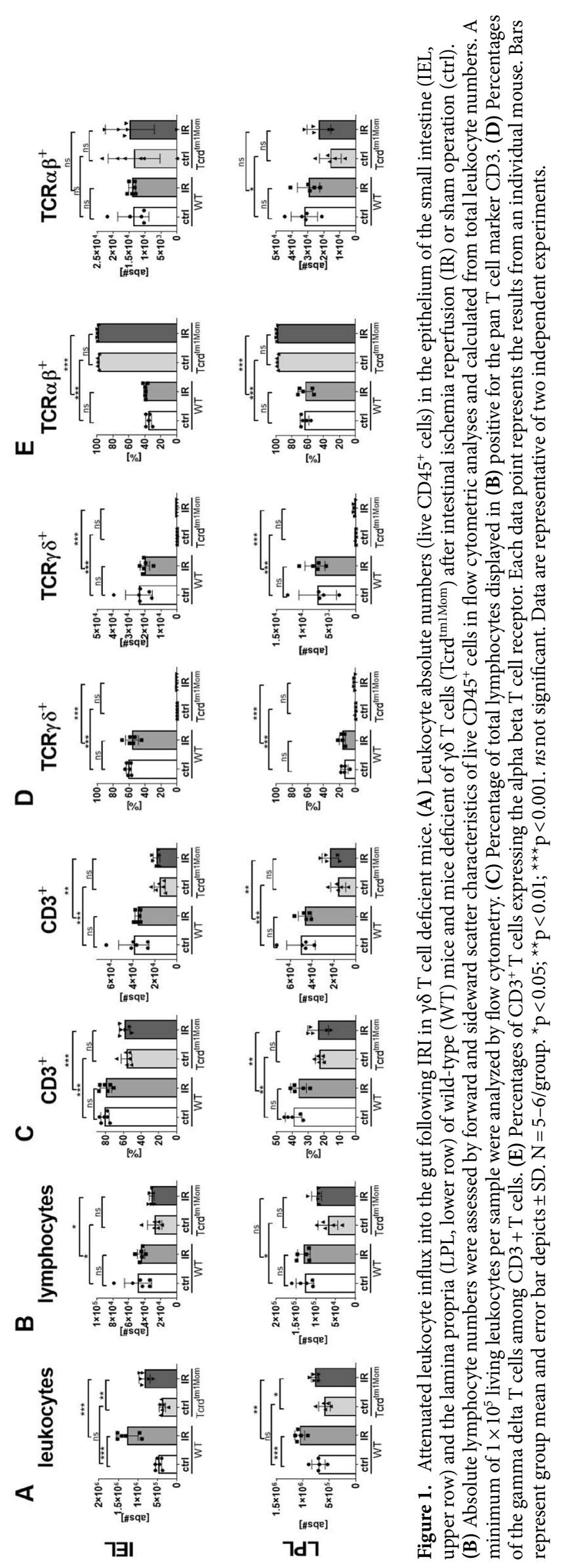


A
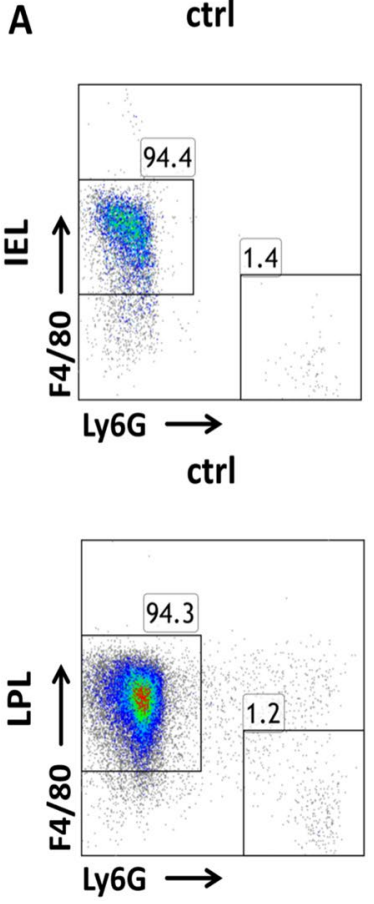
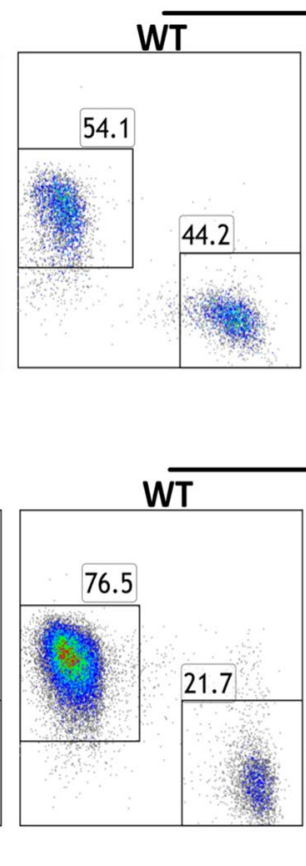

IR

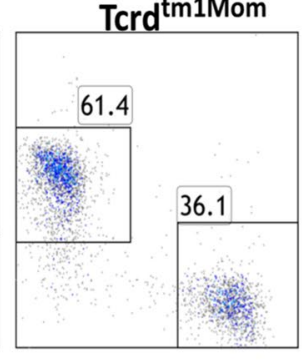

IR
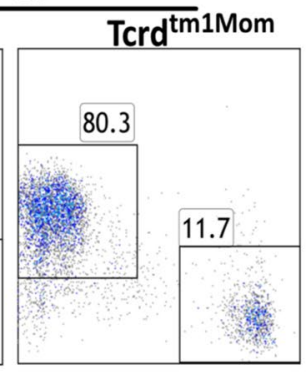

B
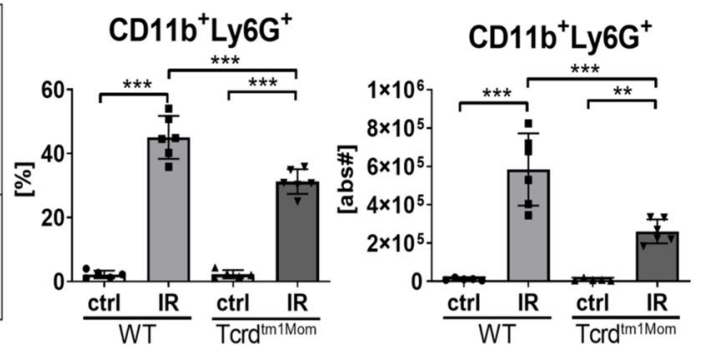

CD11b ${ }^{+}$Ly6G $^{+}$

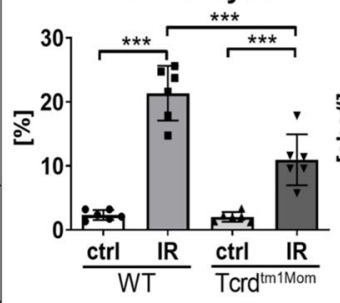

CD11 $\mathrm{b}^{+} \mathrm{Ly}_{6 \mathrm{G}}{ }^{+}$

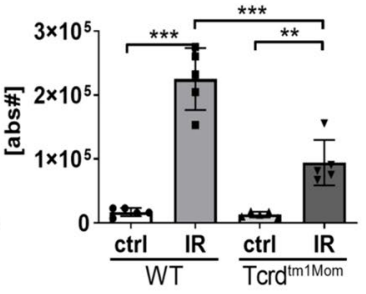

Figure 2. Neutrophil influx into the gut epithelium and lamina propria is reduced in $\gamma \delta \mathrm{T}$ cell deficient mice. Intraepithelial leukocytes (IEL) and lamina propria leukocytes (LPL) were isolated from small intestines and at least $1 \times 10^{5}$ leukocytes per sample analyzed by flow cytometry in wild-type (WT) mice and mice deficient of $\gamma \delta \mathrm{T}$ cells $\left(\mathrm{Tcrd}^{\mathrm{tm} 1 \mathrm{Mom}}\right)$ after intestinal ischemia reperfusion (IR) or sham operation (ctrl). (A) Flow cytometry of live $\mathrm{CD} 45^{+} \mathrm{CD} 11 \mathrm{~b}^{+}$leukocytes in IEL (upper row) and LPL (lower row) showed an increase of Ly6G ${ }^{+}$ neutrophils following IR, which is reduced in $\gamma \delta$ T cell deficient mice compared to WT mice. (B) Percentage of $\mathrm{CD}_{11} \mathrm{~b}^{+}$myeloid cells among IEL (upper graph) and LPL (lower graph) in WT and Tcrd ${ }^{\mathrm{tm} 1 / \mathrm{mom}}$ following IR or ctrl treatment. Each data point represents the result of the analysis of an individual mouse. Bars represent group mean and error bar depicts \pm SD. $N=6$ /group. $n s$ not significant. ${ }^{* * *} \mathrm{p}<0.001$. Data are representative of two independent experiments.

pro-inflammatory cytokines compared to WT mice (Fig. 3). We do not provide direct histological evidence of reduced tissue damage, but show reduced production of $\mathrm{LDH}$ as a marker indicating amelioration of intestinal IRI in mice lacking $\gamma \delta$ T cells (Fig. 4). Besides $\gamma \delta$ T cells, monocytes and macrophages are also capable of the secretion of neutrophil attractants, suggesting a contribution of those cell populations to the neutrophil influx in the post-ischemic gut ${ }^{34}$. We did not formerly address the question whether the lack of $\gamma \delta \mathrm{T}$ cells would influence cytokine production of other T cell fractions. In a previous report, however, we have demonstrated that lack of $\alpha \beta$ T cells does not impact the cytokine profile in the early phases of intestinal IRI ${ }^{9}$.

Reperfusion of the ischemic intestine drives distant organ injury ${ }^{35,36}$, especially acute hepatocellular damage ${ }^{9,37}$. Thus we measured serum levels of transaminases to assess the systemic toxicity of the intestinal inflammatory reaction. Serum levels of transaminases were significantly upregulated after intestinal IR (Fig. 4). Interestingly, in mice lacking $\gamma \delta \mathrm{T}$ cells, the finding of ameliorated local gut inflammation following IRI was accompanied by significantly ameliorated levels of transaminases as compared to wild-type animals (Fig. 4). Confirmation of our results by functionally depleting WT mice of $\gamma \delta$ TCR by mAb treatment would have been desirable in order to eliminate possible confounders as altered gut microbiota in genetically $\gamma \delta$ T cell deficient mice. However, this is not feasible, as mAb treatment does not deplete but generates "invisible" gammadelta $\mathrm{T}$ cells ${ }^{38}$.

Taken together, the key findings of our study are that the genetic deficiency of $\gamma \delta$ T cells in intestinal IRI reduces neutrophil influx, up-regulation of pro-inflammatory cytokines, and the release of transaminases as a marker of distant organ injury (Figs. 1, 2, 3, 4). These findings solve the conflicting results of earlier studies demonstrating amelioration of intestinal IRI in SCID mice, which lack all types of T and B cells ${ }^{7,30,39}$, and our previous study demonstrating that selective $\alpha \beta$ T cells are functionally dispensable for initiating or perpetuating the initial inflammatory reaction ${ }^{9}$., In comparison to other immune cells, $\alpha \beta \mathrm{T}$ cells are relatively rare in the small intestine, whereas $\gamma \delta$ T cells represent the majority of intraepithelial T cells. $\gamma \delta \mathrm{T}$ cells have pleiotropic effector functions, particularly at mucosal borders, including rapid innate-like immune responses ${ }^{40,41}$. Therefore, the isolated deficiency of $\gamma \delta$ T cells in this study offers a feasible explanation for the beneficial effect on acute intestinal IRI. We conclude that in our acute model of intestinal IRI, T cells do, in fact, contribute to the damage, but it is important to differentiate the precise subset and function. $\alpha \beta \mathrm{T}$ cells, which have been unequivocally recognized as key mediators in the pathogenesis of IRI in other organs ${ }^{1,9-11}$, seem to represent innocent bystanders in intestinal IRI. In contrast, $\gamma \delta$ T cells are considered to be more innate T cells, which was here supported by 

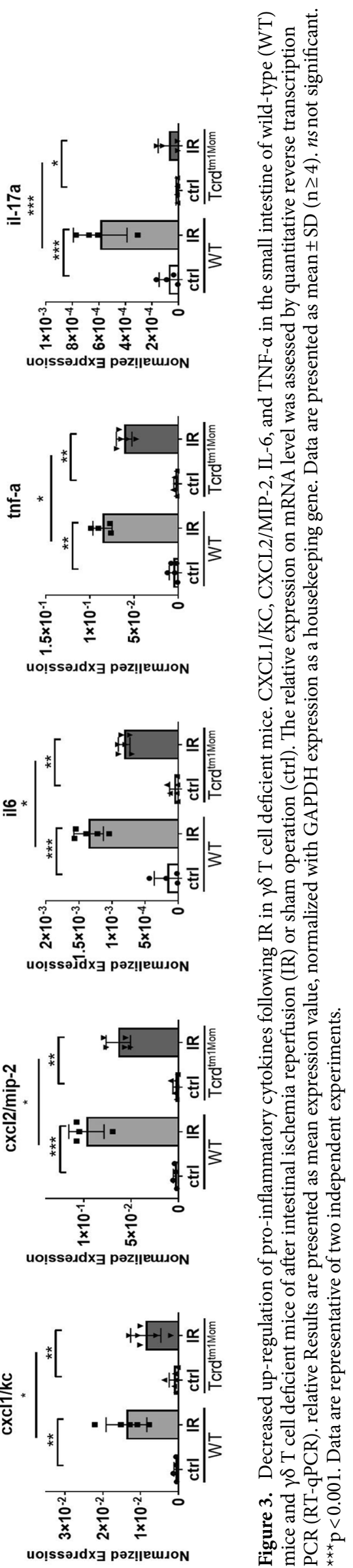

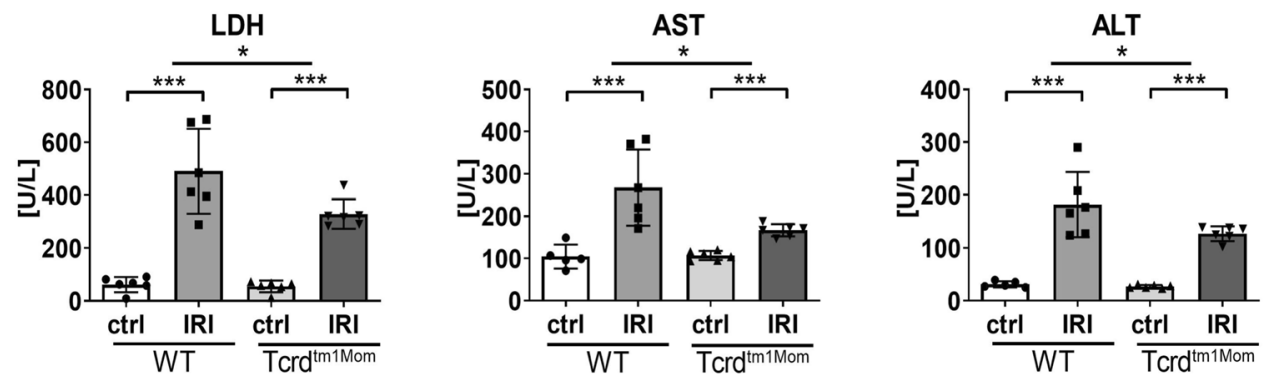

Figure 4. Weaker increase of serum LDH and transaminases following intestinal IRI in $\gamma \delta \mathrm{T}$ cell deficient mice. Levels of serum transaminases AST (left plot) and ALT (right plot) of wild-type (WT) mice and mice deficient of $\gamma \delta \mathrm{T}$ cells $\left(\mathrm{Tcrd}^{\mathrm{tm} 1 \mathrm{Mom}}\right.$ ) after intestinal ischemia reperfusion (IR) or sham operation (ctrl). Each data point represents data of an individual mouse. Each bar represents group mean; error bar depicts SD. N=5 6/group. $n s$ not significant. ${ }^{* *} \mathrm{p}<0.001$. Data are representative of two independent experiments.

their rapid contribution to immune-mediated exacerbation of intestinal IRI. This is in line with recent studies in other models of IRI, demonstrating that $\gamma \delta$ T cells make a significant contribution to IRI ${ }^{17,42-44}$.

In summary, we demonstrate that in the applied model of acute intestinal IR selective $\gamma \delta$ T cell deficiency does significantly ameliorate the severity of the inflammatory response. We conclude that in this model of intestinal IRI, $\gamma \delta$ T cells are critically involved in initiating and perpetuating the initial inflammatory reaction. However, further research is required to clarify the precise role of T cells in intestinal IRI and to better understand the complex interplay between these cells and the other immune cells in this model.

Received: 8 March 2021; Accepted: 29 July 2021

Published online: 20 September 2021

\section{References}

1. Kalogeris, T., Baines, C. P., Krenz, M. \& Korthuis, R. J. Ischemia/reperfusion. Compr. Physiol. 7, 113-170. https://doi.org/10.1002/ cphy.c160006 (2016).

2. Rao, J., Lu, L. \& Zhai, Y. T cells in organ ischemia reperfusion injury. Curr. Opin. Organ Transplant. 19, 115-120. https://doi.org/ 10.1097/MOT.0000000000000064 (2014).

3. Huang, Y., Rabb, H. \& Womer, K. L. Ischemia-reperfusion and immediate T cell responses. Cell Immunol. 248, 4-11. https://doi. org/10.1016/j.cellimm.2007.03.009 (2007).

4. Linfert, D., Chowdhry, T. \& Rabb, H. Lymphocytes and ischemia-reperfusion injury. Transplant Rev. (Orlando). 23, 1-10. https:// doi.org/10.1016/j.trre.2008.08.003 (2009).

5. Hofmann, U. \& Frantz, S. Role of T-cells in myocardial infarction. Eur Heart J. 37, 873-879. https://doi.org/10.1093/eurheartj/ ehv639 (2016).

6. Khandoga, A., Hanschen, M., Kessler, J. S. \& Krombach, F. CD4+ T cells contribute to postischemic liver injury in mice by interacting with sinusoidal endothelium and platelets. Hepatology 43, 306-315. https://doi.org/10.1002/hep.21017 (2006).

7. Shigematsu, T., Wolf, R. E. \& Granger, D. N. T-lymphocytes modulate the microvascular and inflammatory responses to intestinal ischemia-reperfusion. Microcirculation 9, 99-109. https://doi.org/10.1038/sj/mn/7800126 (2002).

8. Watson, M. J. et al. Treatment with antithymocyte globulin ameliorates intestinal ischemia and reperfusion injury in mice. Surgery. 152, 843-850. https://doi.org/10.1016/j.surg.2012.03.001 (2012).

9. Yu, Y. et al. Conventional alpha beta $(\alpha \beta) \mathrm{T}$ cells do not contribute to acute intestinal ischemiareperfusion injury in mice. PLoS ONE 12, 1-15. https://doi.org/10.1371/journal.pone.0181326 (2017).

10. Chien, Y., Meyer, C. \& Bonneville, M. $\gamma \delta$ T cells: First line of defense and beyond. Annu. Rev. Immunol. 32, 121-155. https://doi. org/10.1146/annurev-immunol-032713-120216 (2014).

11. Hayday, A. C. [gamma][delta] cells: A right time and a right place for a conserved third way of protection. Annu. Rev. Immunol. 18, 975-1026. https://doi.org/10.1146/annurev.immunol.18.1.975 (2000).

12. Haas, J. D. et al. Development of interleukin-17-producing $\gamma \delta$ T cells is restricted to a functional embryonic wave. Immunity 37, 48-59. https://doi.org/10.1016/j.immuni.2012.06.003 (2012).

13. Dimova, T. et al. Effector V $\gamma 9 \mathrm{~V} \delta 2 \mathrm{~T}$ cells dominate the human fetal $\gamma \delta$ T-cell repertoire. Proc. Natl. Acad. Sci. U S A. 112, E556E565. https://doi.org/10.1073/pnas.1412058112 (2015).

14. Mowat, A. M. \& Agace, W. W. Regional specialization within the intestinal immune system. Nat. Rev. Immunol. 14, 667-685. https://doi.org/10.1038/nri3738 (2014).

15. Shibata, K. et al. IFN- $\gamma$-producing and IL-17-producing $\gamma \delta$ T cells differentiate at distinct developmental stages in murine fetal thymus. J. Immunol. 192, 2210-2218. https://doi.org/10.4049/jimmunol.1302145 (2014).

16. Havran, W. L. \& Allison, J. P. Origin of Thy-1+ dendritic epidermal cells of adult mice from fetal thymic precursors. Nature 344, 68-70. https://doi.org/10.1038/344068a0 (1990).

17. Shichita, T. et al. Pivotal role of cerebral interleukin-17-producing gammadeltaT cells in the delayed phase of ischemic brain injury. Nat. Med. 15, 946-950. https://doi.org/10.1038/nm.1999 (2009).

18. Gelderblom, M. et al. Neutralization of the IL-17 axis diminishes neutrophil invasion and protects from ischemic stroke. Blood 120, 3793-3802. https://doi.org/10.1182/blood-2012-02-412726 (2012).

19. Itohara, S. et al. T cell receptor delta gene mutant mice: Independent generation of alpha beta $\mathrm{T}$ cells and programmed rearrangements of gamma delta TCR genes. Cell 72, 337-348 (1993).

20. Yu, Y. Y. et al. Increased inflammatory reaction to intestinal ischemia-reperfusion in neonatal versus adult mice. Eur. J. Pediatr. Surg. 25, 46-50. https://doi.org/10.1055/s-0034-1387945 (2015).

21. Klemann, C. et al. Interleukin 17, produced by gd T cells, contributes to hepatic inflammation in a mouse model of BA and is increased in livers of patients. Gastroenterology 150, 229-241.e5. https://doi.org/10.1053/j.gastro.2015.09.008 (2016).

22. Klemann, C. et al. Synthetic retinoid AM80 inhibits Th17 cells and ameliorates experimental autoimmune encephalomyelitis. Am J Pathol. 174, 2234-2245. https://doi.org/10.2353/ajpath.2009.081084 (2009). 
23. Rose, S., Misharin, A. \& Perlman, H. A novel Ly6C/Ly6G-based strategy to analyze the mouse splenic myeloid compartment. Cytometry A. 81, 343-350. https://doi.org/10.1002/cyto.a.22012 (2012).

24. Rong, S. et al. Renal PKC- $\varepsilon$ deficiency attenuates acute kidney injury and ischemic allograft injury via TNF- $\alpha$-dependent inhibition of apoptosis and inflammation. Am. J. Physiol. Renal Physiol. 307, F718-F726. https://doi.org/10.1152/ajprenal.00372.2013 (2014).

25. Ysebaert, D. K. et al. Identification and kinetics of leukocytes after severe ischaemia/reperfusion renal injury. Nephrol. Dial. Transplant. 15, 1562-1574 (2000).

26. Williams, T. M., Wise, A. F., Layton, D. S. \& Ricardo, S. D. Phenotype and influx kinetics of leukocytes and inflammatory cytokine production in kidney ischemia/reperfusion injury. Nephrology (Carlton). https://doi.org/10.1111/nep.12941 (2016).

27. Inagaki-Ohara, K. et al. Mucosal T cells bearing TCRgammadelta play a protective role in intestinal inflammation. J. Immunol. 173, 1390-1398. https://doi.org/10.4049/jimmunol.173.2.1390 (2004).

28. Guy-Grand, D. et al. Two gut intraepithelial CD8+ lymphocyte populations with different T cell receptors: A role for the gut epithelium in T cell differentiation. J. Exp. Med. 173, 471-481. https://doi.org/10.1084/jem.173.2.471 (1991).

29. Qiu, Y. et al. Disturbance of intraepithelial lymphocytes in a murine model of acute intestinal ischemia/reperfusion. J. Mol. Histol. 45, 217-227. https://doi.org/10.1007/s10735-013-9544-1 (2014).

30. Edgerton, C. et al. IL-17 producing CD4+ T cells mediate accelerated ischemia/reperfusion-induced injury in autoimmunity-prone mice. Clin. Immunol. 130, 313-321. https://doi.org/10.1016/j.clim.2008.09.019 (2009).

31. Wang, Z. et al. Autophagy induction ameliorates inflammatory responses in intestinal ischemia-reperfusion through inhibiting NLRP3 inflammasome activation. Shock 19, 52 (2019).

32. Geha, M. et al. IL-17A produced by innate lymphoid cells is essential for intestinal ischemia-reperfusion injury. J. Immunol. 199, 2921-2929. https://doi.org/10.4049/jimmunol.1700655 (2017).

33. Thomas Lee, H. et al. Critical role of interleukin-17A in murine intestinal ischemia-reperfusion injury. Am. J. Physiol.-Gastrointest. Liver Physiol. https://doi.org/10.1152/ajpgi.00201.2012 (2013).

34. Murray, P. J. \& Wynn, T. A. Protective and pathogenic functions of macrophage subsets. Nat. Rev. Immunol. 11, 723-737. https:// doi.org/10.1038/nri3073 (2011).

35. O’Neill, P. J., Cobb, L. M., Steigman, C. K. \& Chaudry, I. H. Prevention of secondary cardiovascular instability after intestinal ischemia and reperfusion improves survival. Am. J. Physiol. 264, R622-R629. https://doi.org/10.1152/ajpregu.1993.264.3.R622 (1993).

36. O'Neill, P. J., Cobb, L. M., Ayala, A., Morrison, M. H. \& Chaudry, I. H. Aggressive fluid resuscitation following intestinal ischemiareperfusion in immature rats prevents metabolic derangements and down regulates interleukin-6 release. Shock 1, 381-387. https:// doi.org/10.1097/00024382-199405000-00012 (1994).

37. Wen, S. et al. HMGB1-associated necroptosis and Kupffer cells M1 polarization underlies remote liver injury induced by intestinal ischemia/reperfusion in rats. FASEB J. https://doi.org/10.1096/f.201900817r (2020).

38. Koenecke, C. et al. In vivo application of mAb directed against the gammadelta TCR does not deplete but generates "invisible" gammadelta T cells. Eur. J. Immunol. 39, 372-379. https://doi.org/10.1002/eji.200838741 (2009).

39. Williams, J.P. et al. Intestinal reperfusion injury is mediated by IgM and complement. J. Appl. Physiol. 86, 938-942. http://www. ncbi.nlm.nih.gov/pubmed/10066708 (1999).

40. Prinz, I., Silva-Santos, B. \& Pennington, D. J. Functional development of $\gamma \delta$ T cells. Eur. J. Immunol. 43, 1988-1994. https://doi. org/10.1002/eji.201343759 (2013).

41. Vantourout, P. \& Hayday, A. Six-of-the-best: Unique contributions of $\gamma \delta$ T cells to immunology. Nat. Rev. Immunol. 13, 88-100. https://doi.org/10.1038/nri3384 (2013).

42. Benakis, C. et al. Commensal microbiota affects ischemic stroke outcome by regulating intestinal $\gamma \delta \mathrm{T}$ cells. Nat. Med. 22, 516-523. https://doi.org/10.1038/nm.4068 (2016).

43. Hochegger, K. et al. Role of /beta and / T cells in renal ischemia-reperfusion injury. AJP Ren. Physiol. 293, F741-F747. https://doi. org/10.1152/ajprenal.00486.2006 (2007).

44. Pai, M.-H., Shih, Y.-M., Shih, J.-M. \& Yeh, C.-L. Glutamine modulates changes in intestinal intraepithelial $\gamma \delta$ T-lymphocyte expressions in mice with ischemia/reperfusion injury. Shock 15, 44 (2015).

\section{Acknowledgements}

It was with shock and disbelief that we learned about the passing away of Faikah Gueler on March 19, 2020. We would like to express our sincere condolences to her children. Your mother was such a wonderful, supportive, enabling, warm-hearted person, and we loved working with her. She will be thoroughly missed. The authors are grateful for excellent technical support by Gertrud Vieten, Stephanie Frömmel, and Herle Chlebusch.

\section{Author contributions}

D.F.: Wrote the manuscript, Conceived and designed the analysis. Y.Y.: Conceived and designed the analysis, Collected the data. X.F.: Collected the data. T.I.: Collected the data. F.G.: Contributed data and analysis tools, Performed the analysis. I.P.: Conceived and designed the analysis, obtained the mice deficient of $\gamma \delta \mathrm{T}$ cells (B6.129P2)-Tcrd $\left.{ }^{t m 1 M o m}\right)$. O.M.S.: Wrote the manuscript, Conceived and designed the analysis. B.U.: Conceived and designed the analysis. J.K.: Conceived and designed the analysis, Collected the data, Contributed data or analysis tools, Performed the analysis, Wrote the manuscript. C.K.: Conceived and designed the analysis, Collected the data, Contributed data or analysis tools, Performed the analysis, Wrote the manuscript. All authors commented on the manuscript.

\section{Funding}

Open Access funding enabled and organized by Projekt DEAL.

\section{Competing interests}

The authors declare no competing interests.

\section{Additional information}

Correspondence and requests for materials should be addressed to C.K.

Reprints and permissions information is available at www.nature.com/reprints.

Publisher's note Springer Nature remains neutral with regard to jurisdictional claims in published maps and institutional affiliations. 
(c) (i) Open Access This article is licensed under a Creative Commons Attribution 4.0 International cc) License, which permits use, sharing, adaptation, distribution and reproduction in any medium or format, as long as you give appropriate credit to the original author(s) and the source, provide a link to the Creative Commons licence, and indicate if changes were made. The images or other third party material in this article are included in the article's Creative Commons licence, unless indicated otherwise in a credit line to the material. If material is not included in the article's Creative Commons licence and your intended use is not permitted by statutory regulation or exceeds the permitted use, you will need to obtain permission directly from the copyright holder. To view a copy of this licence, visit http://creativecommons.org/licenses/by/4.0/.

(C) The Author(s) 2021 